\title{
MicroRNAs in Body Fluids: A More Promising Biomarker for Clear Cell Renal Cell Carcinoma
}

\author{
Lei Shi \\ Mengheng Wang \\ Haiping Li \\ Pengtao You (DD
}

Hubei Key Laboratory of Resources and Chemistry of Chinese Medicine, Hubei University of Chinese Medicine, Wuhan, Hubei, People's Republic of China
Correspondence: Pengtao You Hubei Key Laboratory of Resources and Chemistry of Chinese Medicine, Hubei University of Chinese Medicine, Wuhan, Hubei, People's Republic of China

Tel/Fax +86-27-88920834

Email tptyou@hbtcm.edu.cn

\begin{abstract}
Renal cell carcinoma (RCC) is the second most common cancer of the urinary system, accounting for approximately $10-15 \%$ of kidney cancers in the world. Clear cell renal cell carcinoma (ccRCC) is the most common RCC subtype with the highest mortality. Surgical resection or puncture of tumor tissue is still an important clinical treatment and diagnosis of ccRCC, but its high recurrence rate and poor prognosis often lead to the short survival period of patients. Hence, the development of novel molecular biomarkers is of great clinical importance. miRNAs are endogenous non-coding small RNAs with a length of 19-24 nt. A growing number of studies have reported that miRNAs, as proto-oncogenes or tumor suppressor genes, play a key role in the development of ccRCC and might be effective diagnostic and prognostic biomarkers. In addition, miRNAs can also predict the efficacy of treatment drug, thus improving the accuracy of clinical medication. Furthermore, noninvasive detection of miRNAs or extracellular vesicles (EV) in body fluids has better convenience and repeatability, which shows remarkable advantages compared with tissue detection. In this review, we summarized the typical miRNAs reported in recent years and place emphasis on evaluating miRNAs in different body fluids to provide reference for the clinical diagnosis and prognosis of ccRCC in the future.
\end{abstract}

Keywords: clear cell renal cell carcinoma, microRNA, biomarker, body fluids, exosome

\section{Introduction}

Renal cell carcinoma (RCC) is the second most common cancer in the urological system and accounts for approximately $3 \%$ of global cancer diagnoses and deaths. ${ }^{1}$ An estimated 403,000 people a year are diagnosed with neoplasms of the kidney, of these, approximately 254,500 cases are diagnosed in males and 148,800 in females, reflecting a relative risk (RR) of about 1.7 for men compared to women. ${ }^{2}$ Statistical data showed mortality of 175,000 people from kidney cancer in 2018. This figure constitutes $1.8 \%$ of global cancer deaths. With approximately 114,000 men and 61,000 women perishing from the disease in 2018. ${ }^{3}$ Renal cell carcinoma includes clear cell renal cell carcinoma (ccRCC), papillary renal cell carcinoma (pRCC) and chromophobe renal cell carcinoma (chRCC). Among them, $80 \%$ were ccRCC, which is the most common and aggressive subtype with the highest mortality. ${ }^{4}$ Abundant blood supply to the kidneys drives the early metastasis dissemination of RCC. Approximately $25 \%$ of patients with ccRCC appear hematogenously metastasis to the lungs, liver and bones, and most of them have developed metastasis at the initial diagnosis. ${ }^{5}$ Surgical resection or biopsy of renal tumor tissue is still an important treatment, but its high recurrence rate and poor prognosis remain a challenge. ${ }^{6}$ Tyrosine kinase inhibitors (TKIs), a kind of enzyme receptor 
proteins that could inhibit tumor proliferation, angiogenesis and invasion, is the first-line drug for the treatment of ccRCC over the past 2 decades. However, TKIs only slightly prolong the lives of patients and some patients eventually develop resistance to treatment. ${ }^{7}$ More recently, the emergence of checkpoint inhibitors for RCC, such as PD-1 antibodies, has significantly improved the treatment landscape for patients with RCC. ${ }^{8}$ Its effective rate is superior to traditional targeted therapy drugs, becoming a new first-line treatment drug. ${ }^{9}$ Moreover, a considerable number of ccRCC patients are diagnosed with metastatic disease and some patients have distant metastases during follow-up, so early diagnosis of ccRCC appears to be necessary for effective treatment. ${ }^{10}$ In advanced stages, limited treatment options often lead to poor prognosis. ${ }^{11}$ For this reason, it is of great clinical importance to develop new molecular biomarkers, which can significantly improve the early differential diagnosis and prognosis of renal tumors.

MicroRNAs (miRNAs) are endogenous non-coding small RNAs with a length of about 19-24 nt. They can inhibit or promote the transcription of messenger RNA (mRNA) by targeting the 3'untranslated region (3'UTR) of target gene mRNA, in this way, regulating the level of protein. ${ }^{12}$ More and more evidence showed that miRNAs were closely related to tumors and involved in many biological changes such as proliferation, migration, invasion and apoptosis of tumor cells. ${ }^{13}$ Studies have found that miRNAs can regulate oncogenes and tumor suppressor genes of RCC, which play an important role in the occurrence and development of RCC. ${ }^{13,14}$

Additionally, miRNAs are very stable molecules in body fluids (serum or plasma and urine), thus they may constitute targets for the non-invasive, modern quantification assays, being also considered as potential multifunctional biomarkers in a variety of pathological conditions, including cancer. ${ }^{15}$ miRNAs have a huge potential as efficient prognostic, diagnostic and predictive biomarkers. ${ }^{16}$ Therefore, this review aims to summarize the typical miRNAs reported in recent years and place emphasis on evaluating miRNAs in different body fluids to provide reference for the clinical diagnosis and prognosis of ccRCC in the future.

\section{miRNAs are Related to the Occurrence of ccRCC Mediated by HIF-VHL Signals}

Overproliferation and neovascularization, which consumes a lot of oxygen, is one of the conspicuous features of tumor cells. Hypoxia inducible factor (HIF), a transcription factor with highly conservative and transcriptional activity, is widely existed in human cells under hypoxia. ${ }^{17}$ So far, three HIF-subunits have been identified (HIF- $1 \alpha$, HIF- $2 \alpha$ and HIF-3 $\alpha) .{ }^{18}$ Under normal oxygen partial pressure, the HIF subunit is hydroxylated by proline hydroxylase and recognized by the tumor suppressor gene von Hippel-Lindau (VHL, a substrates recognition component of the E3 ubiquitin linker enzyme), to form the VHL-HIF-E3 complex, which promotes the ubiquitination of HIF- $\alpha{ }^{19}$ Then, the ubiquitinated HIF- $\alpha$ is rapidly degraded by the proteasome, ${ }^{20}$ which keeps intracellular HIF- $\alpha$ at a low level. However, when the cell microenvironment is hypoxic, the function of the tumor suppressor gene VHL is lost ascribed to mutation or deletion, and HIF- $\alpha$ subunit is not degraded into the cell, resulting in the overexpression of HIF. ${ }^{21}$ HIF binds to specific sequences of target genes related to energy metabolism, angiogenesis, and cell growth, and regulates their transcription, thereby promoting cell proliferation, neovascularization and distant metastasis, ultimately leading to hereditary and sporadic RCC. ${ }^{22}$ One of the most important features of RCC is the deletion of VHL gene, which is mainly manifested by mutations, deletions, or hypermethylation of its promoter region. ${ }^{23}$ Inactivating somatic mutations or deletions in the VHL tumor-suppressor gene region (chromosome arm $3 p$ ) are implicated in as many as $45 \%$ of clear cell carcinoma cases. $^{2}$ The overexpression of HIF improves the tolerance of tumor cells to anoxic environment and also increases the expression of vascular endothelial growth factor (VEGF), thus improving the ability of tumor tissue to generate new blood vessels. ${ }^{24}$

Some miRNAs were convinced to be participated the regulation of VHL/HIF signals, thereby affecting the occurrence and development of ccRCC. miR-210, which has been report the most sensitive and influential miRNA regulated by the HIF-1 signals under hypoxia, it's upregulated in ccRCC tissues and targets the ISCU (iron sulphur cluster homologue) 1/2 gene. $^{25-27}$ Furthermore, the expression of VHL mRNA was negatively correlated with miR-210. It should be noted that HIF-2 $\alpha$ also has a small regulatory effect on miR-210 when HIF- $1 \alpha$ is absent. $^{26,28}$ Neal et al found that miR-210 was overexpressed in tumor tissues of patients with ccRCC, and the overall survival of patients was negatively correlated with miR-210. VHL sequencing and methylation-specific PCR (MSP) further demonstrated that miR-210 was associated with VHL mutation and promoter methylation. ${ }^{29}$ More 
than that, the expression of miR-210 was also positively correlative with CAIX (Carbonic Anhydrase IX), which is a transcriptional target of HIF- $1 \alpha$ and a significant marker of hypoxia in tumor cells, ${ }^{30}$ suggesting the regulatory mechanism of miR-210 on hypoxia tolerance in the microenvironment of ccRCC tumor cells through a HIFdependent pattern.

Moreover, in an analysis of the global miRNA profiling in the tumor tissues of VHL-associated patients with ccRCC, a total of 103 miRNAs were identified to be dysregulated, including unsurprising miR-210, miR-155$5 \mathrm{p}, \quad \operatorname{miR}-28-5 \mathrm{p}, \quad \operatorname{miR}-30 \mathrm{c}-3 \mathrm{p}$ and miR-30a-3p. ${ }^{31}$ Previously, Hell et al have proven miR-28-5p can induce the inhibition of Mad2 (Mitotic arrest deficient 2, a mitotic checkpoint protein) translation, which is directly related to the inactivation of VHL. ${ }^{32}$ In another more convincing study, miR-30c-2-3p and miR-30a-3p act as tumor suppressor genes in ccRCC to inhibit the expression of the oncogenic protein HIF-2 $\alpha$ and thereby compensatory increase the level of HIF-1 $\alpha$. In vivo experiments and the predicted survival rate of ccRCC patients were consistent with the above results. ${ }^{33}$ This compensatory mechanism of ccRCCs tumor cells provides a novel idea for the future development of miRNA-based drugs. Wu et al found that miR-21 inhibitors decreased the expression of HIF-1 $\alpha$ mRNA when studying the effect of p-cresyl sulfate (pCS) on HIF and miR-21 in ccRCC. Besides, HIF- $1 \alpha$ and VHL protein expression were inhibited, while HIF-2 $\alpha$ was promoted after treatment with miR-21 inhibitors. ${ }^{34}$ Together, these results imply that miR-21 works in the upstream of HIF-1 $\alpha$, thereby modulating the activities of ccRCC.

miRNAs could also regulate HIF expression in a VHLindependent manner. ${ }^{29}$ miRNAs sequencing of 786-O and hela cells illustrated miR-126-3p and miR-126-5p were both differentially expressed. Gene knockout and luciferase reporting experiments verified that the direct targets of miR-126-3p and miR-126-5p were SLC7A5 and SERPINE1, respectively, which had also been proved the downstream targets of HIF. In the exogenous VHLexpressing 786-O cells, miR-126 gene knockout increased HIF expression without affecting VHL protein expression. The survival time of $516 \mathrm{ccRCC}$ patients from the Cancer Genome Atlas (TCGA) was closely bound up with miR126-3p ( $\mathrm{P}=2.5 \mathrm{E}-04)$ and miR-126-5p ( $\mathrm{P}=1.9 \mathrm{E}-03)$. Of note, the level of miR-126 had a negative time-correlation with clinical stages of the patients with RCC. ${ }^{35}$

$\mathrm{Ma}$ et al detected lower expression of miR-185 in 40 ccRCC clinical samples compared to the adjacent non-tumor tissues, especially in VHL-inactivated tissues. miR-185 also inhibits the proliferation and induces apoptosis of ccRCC tumor cells by targeting VEGFA gene. However, the overexpression of miR-185 had little effect on VHL-intact Caki1 cells. $^{36}$ This is consistent with the report from Kim and Kaelin, that is, VHL-independent manner can lead to the upregulation of VEGF in tumor formation of ccRCC without VHL alterations. ${ }^{37}$ To sum up, miR-185 exerts anti-tumor effect by targeting VEGFA in VHL-inactivated ccRCC cells.

\section{miRNAs in Body Fluids Associated with CCRCC}

Traditional detection methods of RCC, such as puncture or surgery, often bring trauma to patients. Many years of clinical observations have shown that bilateral total nephrectomy can induce or accelerate the growth of VHL tumors in other sites, mainly due to immunosuppressive therapy and dialysis after transplantation. ${ }^{38}$ Moreover, in most cases, surgical methods cannot accurately distinguish between benign and malignant tumors and many patients even must undergo surgical treatment for noncancerous tumors. Non-invasive biomarkers facilitate avoiding unnecessary surgery. Therefore, it is critical to diagnose the disease by non-invasive detection of biomarkers in body fluids. Liquid biopsies, which are minimally invasive, repeatable at multiple time points and helpful to monitor disease progression, can capture biomarkers in urine, serum and plasma. ${ }^{39}$ The clinical application of liquid biopsies to the detection of miRNAs in the body fluids of RCC patients has great potential, which could provide comprehensive information for tumor diagnosis.

Increasing evidence showed that miRNAs played a significant role in $\mathrm{RCC}$, they are reliable as diagnostic markers and stable in circulating body fluids. ${ }^{40}$ Some miRNAs that could act as biomarkers in body fluids-urine or blood for clinical application of RCC will be discussed below.

\section{miRNAs Expression in Urine}

For the first time, the expression level of miRNAs in urine was used for ccRCC studied by von Brandenstein et al, who observed up-regulated miR-15a in the urine of patients with malignant ccRCC. ${ }^{41}$ Similar results were found in another study, in which miR-15a was differentially expressed in urine from a total of 67 patients with RCC and 15 healthy subjects. ${ }^{4}$ Moreover, there was no difference in expression among various subtypes of RCC. Interestingly, miR-15a is a tumor suppressor gene in other 
cancers, such as breast cancer and nasopharyngeal cancer. $^{42,43}$

Li et al verified that the expression of urinary miR-210 was higher in ccRCC patients than control subjects by performing RT-PCR. One week after the operation, the level of urinary miR-210 was remarkably reduced. Receiver operating characteristic (ROC) curve showed an excellent early diagnosis ability for miR-210 with an AUC of 0.76 , a sensitivity of $57.8 \%$ and specificity of $80 \%$. ${ }^{44}$ During follow-up with ccRCC patients after nephrectomy, Petrozza et al detected a significant decrease in urinary miR-210 of patients, while miR-21 and miR-221 showed no significant change. ${ }^{45}$ Subsequently, the authors corroborated this again in urine samples from two larger cohorts, ${ }^{46}$ which emphasized the diagnostic and predictive effects of miR-210 on ccRCC.

For the initial screening phase, 754 miRNAs of urine specimens from renal oncocytoma (benign tumor, generally with good prognosis) and early-stage ccRCC were analyzed using miRNA microarray. ${ }^{47}$ Nine urinary miRNAs were identified, among which miR-432 showed the most remarkable discrimination ability in early-stage ccRCC. But in another independent cohort, urinary miR328 was the only miRNA that could distinguish between progressive and non-progressive ccRCC. In addition, the level of miR-328 was intimately correlated with overall survival (OS) of ccRCC from TCGA, illuminating that urinary miR-328 is a prognostic marker for early-stage ccRCC. $^{48}$

MiR-30a is a tumor suppressor gene in ccRCC, which can promote autophagy and apoptosis of renal tumor cells by downregulating GRP78 and Beclin-1. ${ }^{48,49}$ Recently, Outeiro-Pinho et al elucidated the mechanism that downregulated miR-30a in ccRCC urine samples was associated with the methylation of promoters. ${ }^{50}$ Briefly, analysis of ccRCC patients from TCGA showed that miR-30a expression was negatively correlated with the levels of methylated promoter. In an independent ccRCC cohort, miR-30a was significantly decreased in tumor tissues and urine specimens, while methylated miR-30a was increased in both. However, the results were opposite in normal kidney tissues. ${ }^{50}$ It is the first proposal that the level of methylated miR-30a might be a specific urine biomarker for diagnosing ccRCC. The study shed light on further seeking the reliable biomarker. It is a new strategy to use methylated miRNA as a diagnostic marker for clinical tumors, and its application may not be limited to ccRCC.

\section{miRNAs Expression in Serum or Plasma}

Due to the convenience of sampling, better specificity of detection and higher stability, more studies have been performed on serum or plasma. ${ }^{51}$ Elevated miR-21 and miR-106a were observed in $30 \mathrm{ccRCC}$ patients compared to 30 healthy subjects. Of note, the levels of miR-21 and miR-106a in serum were markedly decreased one month after surgery and ROC results showed that they were strongly feasible as diagnostic tools. ${ }^{52}$ However, in this study, the number of samples under various categories of ccRCC was too small. For example, ccRCC patients at T2 and T3 was only 4 and 1, respectively, which was not convincing that miR-21 and miR-106a could only diagnose patients at $\mathrm{T} 1$. Therefore, it is necessary to carry out large-scale clinical studies in the subsequent experiments.

Huang et al identified differential expressions of miR20b, miR-30a and miR-196a secreted in the serum of ccRCC patients. $^{53}$ The AUC of miR-20b, miR-30a and miR-196a were $0.807,0.766,0.719$ in training set, 0.780, $0.787,0.717$ in validation set, respectively. Interestingly, backward stepwise logistic regression analysis was performed on the combinations of these three miRNAs and the results showed that AUC significantly increased to 0.938 with $92.5 \%$ sensitivity and $80 \%$ specificity, which exhibiting their excellent diagnostic capabilities. Bioinformatics analysis of miR-20b, miR-30a and miR196a of ccRCC patients from TCGA revealed that ITGA4 and NRP2 were target genes of the three miRNAs. ${ }^{53}$ These results indicate that circulating miR-20b, miR-30a and miR-196a in serum play tumor-suppressive roles in vivo. The combined detection of multiple miRNAs shows higher reliability than a single miRNA. Future clinical trials could consider combining multiple miRNAs and using miRNAs clusters as research objectives.

Small RNA expression profiles were constructed in serum of 18 patients with ccRCC and 8 patients with renal benign tumor, in which 29 differentially expressed miRNAs were detected. Subsequently, in an independent cohort, RT-PCR showed that there was no significant difference in serum circulating miR-122-5p and miR-206 levels between 68 ccRCC patients and 47 benign tumor patients, while miR-122-5p and miR-206 were differentially expressed compared with 28 healthy subjects. Furthermore, COX regression analysis manifested that excessive serum miR-122-5p and miR-206 were associated with progression-free, cancer-specific and OS, so they could serve as clinical prognostic indicators. ${ }^{54}$ Unlike 
most studies comparing serum from renal cancer patients and healthy subjects, this study creatively enrolled patients with benign tumors in the analysis cohort containing angiomyolipoma (AML), oncocytoma and complicated kidney cysts. It is a pity that discriminating miRNAs have not been found in the serum of patients with benign tumors, which may be verified in tissue samples.

miR-508-3p and miR-885-5p are the other two novel miRNAs with diagnostic value in ccRCC serum. Liu et al analyzed the differentially expressed miRNAs in ccRCC from Geo and TCGA databases, 4 miRNAs (miR-141-3p, miR-508-3p, miR-885-5p and miR-592) were obtained by taking an intersection. ${ }^{55}$ In a small cohort of ccRCC patients $(n=20)$, the expression levels of miR-141-3p and miR-508-3p were significantly down-regulated, while miR-885-5p and miR-592 were up-regulated. Additionally, the AUC of miR-508-3p and miR-885-5p were the highest. In another large sample $(n=120)$, the combination of miR-508-3p and miR-885-5p showed higher diagnostic reliability (AUC: 0.95; 95\% CI: 0.84 0.96) than miRNA alone. Serum miR-508-3p levels were correlated with $\mathrm{T}$ stage, metastasis, Fuhrman grading and TNM stage, while miR-885-5p was significantly correlated with $\mathrm{T}$ stage and Fuhrman grading.

Zhao et al showed that the expression of miR-625-3p in ccRCC tissues was significantly higher than normal renal tissues, and the high expression of miR-625-3p was associated with the decrease of OS. ${ }^{56}$ However, the results in serum were reversed, which was explained by authors to be related to the selective release of miR-625-3p from tumor cells into body fluids (such as blood). ${ }^{57}$ In vitro experiments, the significantly up-regulated miR-625-3p was found in Caki-1, 786-O cell lines compared with HK-2 cells. Besides, the overexpression of miR-625-3p contribute to the migration and invasion of Caki-1, 786$\mathrm{O}$ cells and inhibited apoptosis. ${ }^{56}$ The retention of miR$625-3 p$ in tumor tissues might promote the deterioration of ccRCC phenotype, which plays a key role in the late metastasis of tumor.

Previous studies have shown that the concentrations of circulating miRNAs in serum and corresponding plasma samples of the same individual is of huge difference, which might result in variant miRNAs with biological functions in serum and plasma. ${ }^{58}$

Detection of 1523 human miRNAs in plasma of 5 ccRCC patients preoperatively and 7 days postoperatively by miRNA microarray showed that miR-144-3p was significantly up-regulated preoperatively. MiR-320c, which did not change between ccRCC and healthy controls, was used as exogenous normalizer, and cel-miR-39 as endogenous normalizer, Lou et al verified the expression of plasma miR-144-3p in 106 ccRCC patients, 28 angiolipoma patients, and 123 healthy subjects by performing qRT-PCR. In both normalized reagents, plasma miR-144$3 p$ levels in the ccRCC group were significantly higher than healthy control group and the angiolipoma group. Moreover, after surgery, no matter partial nephrectomy or radical nephrectomy, the reduction of plasma miR-144-3p could be discovered by using miR-320c or cel-miR-39 as normalizer. $^{59}$ The novel aspect of the study is that the authors used miR-320c as a normalizer for detecting RCC and gave a clear interpret that plasma miR-144-3p is a marker that can distinguish ccRCC from renal angiolipoma with good ability of prognosis. One limitation, however, was that the microarray detected only a small number of plasma specimens.

Chanudet et al constructed a genome-wide miRNA profile based on 754 miRNAs in a large-scale of plasma samples (94 ccRCC patients and 100 healthy controls). However, after multiple testing and corrections for the results, no miRNA was found specifically expressed in ccRCC. Only miR-150 was significantly associated with disease-specific survival and OS $(\mathrm{q}=0.004$ and $\mathrm{q}=0.03$, respectively). ${ }^{60}$ It suggested that plasma miR-150 levels might be helpful to the late-stage prognosis of ccRCC, but further molecular mechanism studies are necessary. In general, their results showed an opposite evidence that plasma miRNAs are not suitable for early detection of ccRCC, while miR-150 was, to some extent, a prognostic indicator.

In addition to miRNAs genome-wide sequencing of plasma samples, more studies have selected candidate miRNAs as a strategy. The lower miR-483-5p was identified in the plasma of ccRCC patients and its expression was significantly reversed 7 days after surgery. Attractively, plasma miR-483-5p levels were negatively correlated with neutrophil-to-lymphocyte ratio (NLR) and lymphocyte-to-monocyte ratio (LMR), ${ }^{61}$ which has been previously reported important prognostic factors related to inflammatory response in patients with ccRCC. ${ }^{62,63}$ In vitro, miR-483-5p mimic upregulated the expression of E-cadherin protein and downregulated $\mathrm{N}$-cadherin protein, suggesting that miR-483-5p is involved in the epithelial mesenchymal transition (EMT) of renal cancer cells. ${ }^{61}$ The authors attempted to reveal a mechanism that miR-483-5p 
reversed EMT of ccRCC by resisting inflammation in peripheral blood of renal cancer cells, thus playing an anticarcinogenic role. However, more evidence is needed to clarify whether miR-483-5p plays a decisive role and directly participates in this process.

Proteolipid protein 2 (PLP2), a protein involved in lipid metabolism, whose elevation often leads to abnormal lipid accumulation. ${ }^{64}$ Nowadays, convincing evidence emerged that plasma miRNA and PLP2 are associated with ccRCC. Xiao et al found that the expression of plasma miR-765 in ccRCC was significantly lower than the non-cancer group, and PLP2 gene was the direct target of miR-765. Both miR-765 mimic and PLP2 siRNA inhibited the proliferation, invasion and lipid accumulation of various renal cell lines, while it was reversed after cotransfection of miR-765 mimic and PLP2. ${ }^{65}$ The results indicated that miR-765 inhibited the worse progression of renal cancer cells and promoted lipid clearance by directly targeting PLP2. Consequently, reduced plasma miR-765 turned out to be an excellent detection target.

\section{Comparison with Tissues and Plasma}

Chen et al performed RT-PCR to detect tissues and the matched plasma levels of miR-210, miR-224, miR-141 in ccRCC, as well as evaluating their diagnostic accuracy. Especially, ROC analysis showed that the miR-224/miR141 ratio in tissues had significant diagnostic reliability, while plasma miR-210 and miR-224 were not associated with the diagnosis of ccRCC. ${ }^{66}$ Doubts about the diagnostic ability of miRNAs in plasma are not uncommon, Chanudet et al have reached similar conclusions earlier. ${ }^{60}$ Currently, no unified standard for the selection of PCR normalizer for miRNA detection contributed to different selection of normalizer, thereby contributed to variety of results.

\section{miRNAs Expression in Exosomes}

Exosomes, a magical nanovesicles secreted by many types of cells, have a size ranging from 50 to $150 \mathrm{~nm}$ in diameter. ${ }^{67,68}$ Exosomes contain a variety of genetic materials and bioactive molecules, such as DNA, RNA and proteins, which play an important role in intercellular communication of tumor microenvironment. ${ }^{69-71}$ MiRNAs encapsulated in exosomes of tumor tissues could be transported to the outside of cells, resulting in corresponding content changes of miRNAs in body fluid, which drives the metastasis of tumor cells and ultimately death. ${ }^{72,73}$ As a new kind of cancer biomarker, exosomes have attracted much attention in recent years. Accumulating exosomal miRNAs were found to be closely related to ccRCC and might be a promising potential therapeutic target. $^{74}$

miRNAs in urinary exosomes of 46 participants were detected by miRNA array, Butz et al found that miR-126$3 \mathrm{p}$ was significantly down-regulated (5.05-fold change) in 28 ccRCC patients compared to 18 healthy participants. The results of ROC analysis and RT-qPCR validation in another 138 participants (81 ccRCC patients, 24 patients with benign kidney tumor, and 33 healthy participants) showed that pairwise combinations of miR-126$3 p$, miR-34b-5p, miR-150-5p, miR-449a and miR-486-5p had the best sensitivity compared with single one. In addition, the five exosomal miRNAs could also be detected in the medium of primary 786-O, metastatic ACHN and Caki-2 cell lines. The exosomes secreted by 786-O cells were transferred to $\mathrm{ACHN}$, Caki-2 and human umbilical vein endothelial cells (HUVEC). After 24 hours, the internalization of exosomal miRNAs was observed by electron microscopy. ${ }^{75}$ In summary, miR126-3p, miR-34b-5p, miR-150-5p, miR-449a and miR486-5p are potential predictive targets for ccRCC. Exosomal miRNAs play a critical role in the communication between ccRCC and vascular endothelial cells, which might be related to the angiogenesis of renal cancer tumors. $^{76}$

Elevated miR-210 and miR-1233 in serum circulating exosomes of ccRCC patients was observed by Zhang et al for the first time. ${ }^{77}$ Moreover, there was no significant correlation of exosomal miRNA levels with gender, age and ccRCC grade. But after tumor resection, the levels of miR-210 and miR-1233 were significantly higher than those before resection. ROC analysis showed that miR210 (AUC: 0.69; sensitivity: 70\%; specificity: 62.2\%) and miR-1233 (AUC: 0.82; sensitivity: 81\%; specificity: 76\%) were highly feasible as diagnostic markers. Taken together, the above results suggest that the two serum exosomal miRNAs are superior biomarkers for liquid biopsies. Of note, Zhang et al revealed that miR-210 and miR-1233 were upregulated in ccRCC independently of clinical staging and were stable in serum. ${ }^{77}$ Further experiment combining miR-210 and miR-1233 (miR-210/miR1233 ratio) are proposed, but there is still a long way to go before clinical application.

The study of miR-210 goes beyond that. Similarly, Wang et al also found that the serum exosomal miR-210 
was significantly up-regulated in ccRCC. ${ }^{40}$ The levels of miR-210 was not related to gender and age, but its expression was higher in patients with T3/T4 tumor stages and Fuhrman grade III/IV. More importantly, HK-2 cells stimulated by $\mathrm{CoCl}_{2}$ (a hypoxic inducer) released more exosomes that contained higher expression of miR-210. Furthermore, the expression of exosomal miR-210 was also upregulated when this stimulation was applied to the 786-O and SN12-PM6 cell lines. Vacuole membrane protein 1 (VMP1), a $46 \mathrm{kDa}$ tumor suppressor gene closely associated with tumorigenesis, could induce autophagy and apoptosis in RCC. ${ }^{78,79}$ Lower VMP1 expression was found in both HK-2, 786-O and SN12-PM6 cells in a dose-dependent manner after treatment with $\mathrm{CoCl}_{2}{ }^{40} \mathrm{It}$ provides strong evidence that hypoxia induces tumor cells to secrete exosomes into serum, which might be mediated by HIF-VHL signals.

Recently, Crentil et al showed that miR-205 was significantly downregulated (10,000-fold) in 786-O cells compared with HK-2 cells, and this differential expression was consistent with the expression of exosomal miR-205 in supernatant. ${ }^{80}$ The result indicated that extraction of exosomes and determination of miR-205 could distinguish between 786-O and HK-2 cells, but the limitation was that the content of exosomal miR-205 secreted by ccRCC tumors in vivo and its concentration in specific body fluids such as serum and urine were not necessarily the same as the relationship between the content of miR-205 in the medium of cultured cells. Owing to the complexity of the vivo environment, tumor secreting exosomes containing miRNAs is a multi-factor and multi-target process. However, another study demonstrated non-exosomal miR-205 was significantly decreased in clinical ccRCC patients. $^{81}$

Cancer stem cells (CSCs) are a group of selfreplenishing cells in tumor cells and contribute to cancer progression, metastasis, and recurrence, ${ }^{82}$ which could be managed and controlled by miRNAs. ${ }^{83}$ CSCs were isolated from a total of 209 ccRCC patients (133 at stages I/II and 76 at stages III/IV), Wang et al elucidate that miR$19 b-3 p$ contained in exosomal ccRCC stem cells promoted epithelial-mesenchymal transition (EMT) by targeting the gene of phosphatase and tensin homolog deleted on chromosome ten (PTEN). ${ }^{84}$ When treatment with miR-19b-3p inhibitor or knockout of endogenous exosomal miR-19b$3 p$, it is obvious that migration, invasion and proliferation of ccRCC tumor cells are greatly reduced, and EMT- related gene expression is also decreased. The results indicated that the exosomal miR-19b-3p of CSCs was another predictor of advanced metastasis of ccRCC.

In contrast, Song et al certificated in vitro and vivo that exosomal miR-30c-5p was downregulated in ccRCC and may act as a diagnostic target for patients with early-stage ccRCC. ${ }^{85}$ Interestingly, the authors found that miR-30c-5p was not differentially expressed in exosomes of prostate and bladder cancer compared with healthy participants, but significantly reduced in urine of patients with ccRCC (ROC sensitivity: 68.57\%, specificity: 100\%). Luciferase reporter assay confirmed that miR-30c-5p inhibited the expression of heat-shock protein 5 (HSPA5) by binding to the target site in 3 '-UTRs, indicating the direct target of miR-30c-5p was HSPA5. ${ }^{85}$ HSPA5 can protect kidney tumor cells from $\mathrm{T}$ cell killing, promote tumor formation and develop drug resistance. ${ }^{86}$ Due to the lack of effective indicators for early-stage diagnosis in ccRCC, Song et al research on miR-30c-5p-HSPA5 signals is of great potentiality, which provides a new strategy for the future prevention of ccRCC.

Another study performed RNA sequencing on serum samples to construct exosomal miRNA profiles. ${ }^{87}$ The number of miRNAs less than 1000 from the differentially expressed genes was selected for qPCR validation in another 38 subjects ( 22 ccRCC patients and 16 healthy individuals) and only 3 miRNAs were found to be remarkably dysregulated (miR-92a-1-5p, miR-424-3p and miR149-3p). ROC curve analysis showed that AUC, specificity and sensitivity of the three exosomal miRNAs were follows: miR-92a-1-5p: 0.8324, 87.5\%, 77.3\%; miR-424-3p: 0.7727, 75.0\%, 81.8\%; miR-149-3p: 0.7188, 75\%, 72.7\%, respectively. ${ }^{87}$ Previous studies have reported that miR92a-1 played a key role in promoting proliferation, invasion, and migration of liver cancer, ${ }^{88}$ breast cancer, ${ }^{89}$ and colorectal cancer cells. ${ }^{90}$ MiR-424 acts as an oncogene in esophageal squamous carcinoma and thyroid cancer cells. ${ }^{91,92}$ In addition, Jin et al are the first to demonstrate the inhibitory effect of miR-149 on RCC tissues. ${ }^{93}$ Guo et al found that miR-149 strongly inhibited the growth of nonsmall cell lung cancer (NSCLC) and promoted its apoptosis. ${ }^{94}$ However, Xiao et al gave a different evidence that exosomal miR-92a-1-5p was down-regulated while exosomal miR-424-3p and miR-149-3p were significantly up-regulated in $\mathrm{RCC}^{87}$ MiRNAs could play an opposite role in different cancers, but it is thought-provoking that there are differences in expression patterns between exosomes and cell lines of the same cancer. Subsequent clinical 
Table I Classical and Recently Reported miRNAs That Have Potential to Be Biomarkers for RCC

\begin{tabular}{|c|c|c|c|c|}
\hline miRNA Names & Expression Changes in RCC & Target Genes & Detection Site & Reference \\
\hline miR-9-5p & Up-regulate & - & Tissue & {$[100]$} \\
\hline miR-I5a & Up-regulate & - & Urine & {$[4]$} \\
\hline$m i R-19 b-3 p$ & Up-regulate & PTEN & Exosome & [84] \\
\hline miR-20b & Down-regulate & ITGA4, NRP2 & Serum & [53] \\
\hline miR-2I & Up-regulate & PTEN, p53, PDCD4, PIK3RI & Tissue, serum & {$[34,52,105]$} \\
\hline miR-28-5p & Down-regulate & $\operatorname{Mad} 2$ & Tissue & [32] \\
\hline miR-30a-3p & Down-regulate or up-regulate & GRP78, Beclin-I, ITGA4, NRP2 & Tissue, urine, serum & {$[33,50,53]$} \\
\hline $\mathrm{miR}-30 \mathrm{c}$ & Down-regulate & HSPA5 & Tissue, exosome & {$[33,85]$} \\
\hline miR-34b-5p & Up-regulate & - & Exosome & {$[75]$} \\
\hline miR-92a-I-5p & Down-regulate & - & Exosome & [87] \\
\hline miR-I06a & Up-regulate & - & Serum & {$[52]$} \\
\hline miR-I22-5p & Down-regulate or up-regulate & FOXO3 & Serum, tissue & {$[54,97]$} \\
\hline miR-126 & Down-regulate & SLC7A5, SERPINEI & Tissue, exosome & {$[35,75]$} \\
\hline miR-144-3p & Up-regulate & MAP3K8 & Plasma & {$[59,106]$} \\
\hline miR-149-3p & Up-regulate & - & Exosome & [87] \\
\hline miR-I50-5p & Down-regulate or up-regulate & - & Plasma, exosome & {$[60,75]$} \\
\hline miR-154-5p & Up-regulate & - & Tissue & [98] \\
\hline miR-155-5p & Down-regulate & - & Tissue & [99] \\
\hline miR-185 & Down-regulate & VEGFA & Tissue & [36] \\
\hline miR-196a & Down-regulate & ITGA4, NRP2 & Serum & [53] \\
\hline miR-205 & Down-regulate & - & Exosome & [80] \\
\hline miR-206 & Down-regulate & - & Serum & {$[54]$} \\
\hline miR-2I0 & Up-regulate & ISCU I/2 & Tissue, urine, exosome & {$[26,29,40,44,46,77]$} \\
\hline miR-224/miR-I4I & Up-regulate & HS6ST2, LOX & Tissue & {$[66,107]$} \\
\hline $\operatorname{miR}-328$ & Down-regulate & - & Urine & [47] \\
\hline$m i R-424-3 p$ & Up-regulate & WEEI & Exosome & {$[87,108]$} \\
\hline miR-432 & Up-regulate & - & Urine & [47] \\
\hline $\operatorname{miR}-449 a$ & Down-regulate & - & Exosome & [75] \\
\hline$m i R-483-5 p$ & Down-regulate & - & Plasma & {$[6 I]$} \\
\hline miR-486-5p & Down-regulate & TAKI & Exosome & {$[75,109]$} \\
\hline miR-508-3p & Down-regulate & - & Serum & [55] \\
\hline miR-625-3p & Down-regulate or up-regulate & - & Tissue, serum & [56] \\
\hline miR-765 & Down-regulate & PLP2 & Plasma & [65] \\
\hline miR-885-5p & Up-regulate & PLIN3 & Serum & {$[55,110]$} \\
\hline miR-I233 & Up-regulate & - & Exosome & [77] \\
\hline
\end{tabular}

studies of exosomal miRNA should be combined with vitro experiments to illustrate the reliability of miRNA more convincingly as a biomarker in enough cases.

\section{Clinical Value of miRNAs}

There are two strategies for tumor therapy based on miRNA: silence the carcinogenic miRNAs or restore the antitumor miRNAs. Therefore, elevated, or reduced miRNAs in vivo are also potential therapeutic targets. Currently, dysregulated miRNAs in RCC and normal specimens have been observed many times. These miRNAs act as oncogenes or tumor suppressor genes with remarkable diagnosis and discrimination effect in ccRCC. For instance, Youssef et al selected a set of dysregulated miRNAs in 94 cases of different RCC subtypes based on the results of miRNA microarray. In their top scoring pairs classifier system, these miRNAs could splendidly distinguish ccRCC, pRCC and chRCC (sensitivity: 100\%, 97\%, $100 \%$, respectively). ${ }^{95}$ Different miRNA profiles were found in different stages of ccRCC by Qi et al, among which three miRNAs (miR-20, miR-484, miR-497) showed great discriminative ability at stage I. $^{96}$ Moreover, plasma miR-144-3p in ccRCC is significantly higher than angiolipoma and might be a potential tool for identifying benign from malignant tumors. ${ }^{59}$ Certain studies indicated that miRNA expression patterns also have 
prognostic function in renal cancer. miR-122 serve as an oncogene by targeting Forkhead box O 3 (FOXO3), which is significantly increased in ccRCC tissues and associated with shortened patient survival. In addition, its mimics promoted the proliferation, migration and invasion of 786-O cells. ${ }^{97}$ Lin et al demonstrated that up-regulated miR-154 was also a biomarker for poor prognosis in ccRCC. ${ }^{98}$ MiR-155-5p and miR-210-3p were elevated in the patients with recurrent $\mathrm{ccRCC}$, thus indicating that they are predictive for the recurrence of ccRCC. ${ }^{99}$

Recently, accumulating evidence showed that miRNAs were effective biomarkers for predicting the therapeutic effect of TKIs or immunotherapy drug. Assessing the levels of miRNA in patients before medication could improve the accuracy of clinical medication and help clinicians make better decisions. Ralla et al observed elevated expression of miR-9-5p in tissues of ccRCC patients who did not respond to sunitinib treatment, suggesting it was a reliable predictor for drug resistance of sunitinib treatment. ${ }^{100}$ In another study, four miRNAs were identified to be differentially expressed in patients who extreme response to TKIs, in which up-regulated miR-425-5p and down-regulated miR139-3p, let-7d, let-7e were significantly correlated with poor response to TKIs. ${ }^{101}$ By miRNA expression profile analysis, Incorvaia et al identified combinatorial miRNAs of peripheral lymphocytes, especially the miRNAs 22/24 were inversely associated with plasma PD-1 and PD-L1 levels in long-responder patients to nivolumab treatment, showing that miRNAs represent an additional level of regulation of immune checkpoint expression. ${ }^{102}$ Additionally, Qu et al found PD-L1 was a direct target of miR-497-5p in two RCC cell lines, which could help identify responders from patient populations. ${ }^{103}$

\section{Conclusions}

In conclusion, an increasing body of evidence suggests that miRNAs are reliable ccRCC biomarkers. In this article, we reviewed miRNAs in different environments of ccRCC tumor cell and critically evaluated their clinical applicability (the miRNAs mentioned above are shown in Table 1). miRNAs have unique advantages in early diagnosis, differentiation of subtypes and stages, and advanced-stage prognosis of ccRCC. Especially, noninvasive detection of miRNAs in body fluids has great molecular stability. Moreover, miRNAs can distinguish between benign and malignant renal tumors, as well as distant metastases and non-metastatic tumors. ${ }^{104}$
However, there are still some problems to be solved in the research field of miRNA, such as why do mutation occur in the VHL gene, whether there is uniformity of miRNA expression in patients of different regions. Poor tissue-specific targeting, off-target effects and the low safety also need to overcome if developing miRNA therapy. Mode of action and regulatory mechanism of miRNA in renal cells still need to be further explored, which will provide a solid foundation for targeted therapy of tumors. Existing studies have revealed dozens of miRNAs with potential biomarkers and screening miRNAs with the most clinically value from them will be a challenge. However, due to the numerous target genes of miRNA and the complex regulatory mechanism, the accuracy of single miRNA as a tumor biomarker is limited. Hence, the panel miRNAs or the combination of miRNA detection and other diagnostic methods, such as advanced imaging techniques or biopsies methods might be a better strategy. It is promising to find reliable miRNA diagnostic markers and develop therapeutic drugs based on targeted miRNAs.

\section{Acknowledgments}

Thanks to all those who have contributed to this manuscript. No external funding was used in the preparation of this manuscript.

\section{Disclosure}

All the authors declare that they have no conflicts of interest in this work.

\section{References}

1. Rini BI, Campbell SC, Escudier B. Renal cell carcinoma. Lancet. 2009;373(9669):1119-1132. doi:10.1016/S0140-6736(09)60229-4

2. Padala SA, Barsouk A, Thandra KC, et al. Epidemiology of renal cell carcinoma. World J Oncol. 2020;11(3):79-87. doi:10.14740/ wjon 1279

3. Bray F, Ferlay J, Soerjomataram I, et al. Global cancer statistics 2018: GLOBOCAN estimates of incidence and mortality worldwide for 36 cancers in 185 countries. CA Cancer J Clin. 2018;68(6):394-424. doi:10.3322/caac.21492

4. Mytsyk Y, Dosenko V, Borys Y, et al. MicroRNA-15a expression measured in urine samples as a potential biomarker of renal cell carcinoma. Int Urol Nephrol. 2018;50(5):851-859. doi:10.1007/ s11255-018-1841-x

5. Aguiari G. MicroRNAs in clear cell renal cell carcinoma: biological functions and applications. J Kidney Cancer VHL. 2015;2(4):140-152. doi:10.15586/jkcvhl.2015.40

6. Jonasch E, Gao J, Rathmell WK. Renal cell carcinoma. BMJ. 2014;349(nov10 11):g4797. doi:10.1136/bmj.g4797

7. Rini BI, Atkins MB. Resistance to targeted therapy in renal-cell carcinoma. Lancet Oncol. 2009;10(10):992-1000. doi:10.1016/ S1470-2045(09)70240-2 
8. Bedke J, Albiges L, Capitanio U, et al. The 2021 updated European Association of Urology guidelines on renal cell carcinoma: immune checkpoint inhibitor-based combination therapies for treatment-naive metastatic clear-cell renal cell carcinoma are standard of care. Eur Urol. 2021;80(4):393-397. doi:10.1016/j. eururo.2021.04.042

9. Labriola MK, Batich KA, Zhu J, et al. Immunotherapy is changing first-line treatment of metastatic renal-cell carcinoma. Clin Genitourin Cancer. 2019;17(3):e513-e521. doi:10.1016/j. clgc.2019.01.017

10. Crispen PL, Breau RH, Allmer C, et al. Lymph node dissection at the time of radical nephrectomy for high-risk clear cell renal cell carcinoma: indications and recommendations for surgical templates. Eur Urol. 2011;59(1):18-23. doi:10.1016/j. eururo.2010.08.042

11. Motzer RJ, Russo P. Systemic therapy for renal cell carcinoma. $J$ Urol. 2000;163(2):408-417. doi:10.1016/S0022-5347(05) 67889-5

12. Bartel DP. MicroRNAs: target recognition and regulatory functions. Cell. 2009;136(2):215-233. doi:10.1016/j.cell.2009.01.002

13. Volinia S, Calin GA, Liu CG, et al. A microRNA expression signature of human solid tumors defines cancer gene targets. Proc Natl Acad Sci $U$ S A. 2006;103(7):2257-2261. doi:10.1073/pnas.0510565103

14. Heinzelmann J, Henning B, Sanjmyatav J, et al. Specific miRNA signatures are associated with metastasis and poor prognosis in clear cell renal cell carcinoma. World J Urol. 2011;29 (3):367-373. doi:10.1007/s00345-010-0633-4

15. Kosaka N, Iguchi H, Ochiya T. Circulating microRNA in body fluid: a new potential biomarker for cancer diagnosis and prognosis. Cancer Sci. 2010;101(10):2087-2092. doi:10.1111/ j.1349-7006.2010.01650.x

16. Cho WC. MicroRNAs: potential biomarkers for cancer diagnosis, prognosis and targets for therapy. Int J Biochem Cell Biol. 2010;42(8):1273-1281. doi:10.1016/j.biocel.2009.12.014

17. Walmsley SR, McGovern NN, Whyte MK, et al. The HIF/VHL pathway: from oxygen sensing to innate immunity. Am J Respir Cell Mol Biol. 2008;38(3):251-255. doi:10.1165/rcmb.2007-0331TR

18. Gu YZ, Moran SM, Hogenesch JB, et al. Molecular characterization and chromosomal localization of a third alpha-class hypoxia inducible factor subunit, HIF3alpha. Gene Expr. 1998;7 (3):205-213.

19. Yu F, White SB, Zhao Q, et al. Dynamic, site-specific interaction of hypoxia-inducible factor-1alpha with the von Hippel-Lindau tumor suppressor protein. Cancer Res. 2001;61(10):4136-4142.

20. Jaakkola P, Mole DR, Tian YM, et al. Targeting of HIF-alpha to the von Hippel-Lindau ubiquitylation complex by O2-regulated prolyl hydroxylation. Science. 2001;292(5516):468-472. doi:10.1126/science. 1059796

21. Ivan $\mathrm{M}$, Kondo $\mathrm{K}$, Yang $\mathrm{H}$, et al. HIFalpha targeted for VHL-mediated destruction by proline hydroxylation: implications for O2 sensing. Science. 2001;292(5516):464-468. doi:10.1126/ science. 1059817

22. Banumathy G, Cairns P. Signaling pathways in renal cell carcinoma. Cancer Biol Ther. 2010;10(7):658-664. doi:10.4161/ cbt.10.7.13247

23. Kaelin WG Jr. The von Hippel-Lindau tumor suppressor protein and clear cell renal carcinoma. Clin Cancer Res. 2007;13(2 Pt 2):680s-684s. doi:10.1158/1078-0432.CCR-06-1865

24. Gossage L, Eisen T. Alterations in VHL as potential biomarkers in renal-cell carcinoma. Nat Rev Clin Oncol. 2010;7(5):277-288. doi:10.1038/nrclinonc.2010.42

25. Huang WC, Swietach P, Vaughan-Jones RD, et al. Differentiation impairs low $\mathrm{pH}$-induced $\mathrm{Ca} 2+$ signaling and ERK phosphorylation in granule precursor tumour cells. Cell Calcium. 2009;45 (4):391-399. doi:10.1016/j.ceca.2009.01.002
26. McCormick RI, Blick C, Ragoussis J, et al. miR-210 is a target of hypoxia-inducible factors 1 and 2 in renal cancer, regulates ISCU and correlates with good prognosis. Br J Cancer. 2013;108 (5):1133-1142. doi:10.1038/bjc.2013.56

27. Fasanaro P, Greco S, Lorenzi M, et al. An integrated approach for experimental target identification of hypoxia-induced miR-210. $J$ Biol Chem. 2009;284(50):35134-35143. doi:10.1074/jbc. M109.052779

28. Fedorko M, Pacik D, Wasserbauer R, et al. MicroRNAs in the pathogenesis of renal cell carcinoma and their diagnostic and prognostic utility as cancer biomarkers. Int J Biol Markers. 2016;31(1):e26-37. doi:10.5301/jbm.5000174

29. Neal CS, Michael MZ, Rawlings LH, et al. The VHL-dependent regulation of microRNAs in renal cancer. BMC Med. 2010;8 (1):64. doi:10.1186/1741-7015-8-64

30. Loncaster JA, Harris AL, Davidson SE, et al. Carbonic anhydrase (CA IX) expression, a potential new intrinsic marker of hypoxia: correlations with tumor oxygen measurements and prognosis in locally advanced carcinoma of the cervix. Cancer Res. 2001;61 (17):6394-6399.

31. Gattolliat $\mathrm{CH}$, Couve $\mathrm{S}$, Meurice $\mathrm{G}$, et al. Integrative analysis of dysregulated microRNAs and mRNAs in multiple recurrent synchronized renal tumors from patients with von Hippel-Lindau disease. Int $J$ Oncol. 2018;53(4):1455-1468. doi:10.3892/ ijo. 2018.4490

32. Hell MP, Thoma CR, Fankhauser N, et al. miR-28-5p promotes chromosomal instability in VHL-associated cancers by inhibiting Mad2 translation. Cancer Res. 2014;74(9):2432-2443. doi:10.1158/0008-5472.CAN-13-2041

33. Mathew LK, Lee SS, Skuli N, et al. Restricted expression of miR-30c-2-3p and miR-30a-3p in clear cell renal cell carcinomas enhances HIF2alpha activity. Cancer Discov. 2014;4(1):53-60. doi:10.1158/2159-8290.CD-13-0291

34. Wu TK, Wei CW, Pan YR, et al. The uremic toxin p-cresyl sulfate induces proliferation and migration of clear cell renal cell carcinoma via microRNA-21/ HIF-1alpha axis signals. Sci Rep. 2019;9(1):3207. doi:10.1038/s41598-019-3 9646-9

35. Liu W, Chen $\mathrm{H}$, Wong $\mathrm{N}$, et al. Pseudohypoxia induced by miR-126 deactivation promotes migration and therapeutic resistance in renal cell carcinoma. Cancer Lett. 2017;394:65-75. doi:10.1016/j.canlet.2017.02.025

36. Ma X, Shen D, Li H, et al. MicroRNA-185 inhibits cell proliferation and induces cell apoptosis by targeting VEGFA directly in von Hippel-Lindau-inactivated clear cell renal cell carcinoma. Urol Oncol. 2015;33(4):169 e161-111. doi:10.1016/j. urolonc.2015.01.003

37. Kim WY, Kaelin WG. Role of VHL gene mutation in human cancer. J Clin Oncol. 2004;22(24):4991-5004. doi:10.1200/ JCO.2004.05.061

38. Solomon D, Schwartz A. Renal pathology in von Hippel-Lindau disease. Hum Pathol. 1988;19(9):1072-1079. doi:10.1016/s00468177(88)80089-3

39. Heitzer E, Haque IS, Roberts CES, et al. Current and future perspectives of liquid biopsies in genomics-driven oncology. Nat Rev Genet. 2019;20(2):71-88. doi:10.1038/s41576-0180071-5

40. Wang $\mathrm{X}$, Wang $\mathrm{T}$, Chen $\mathrm{C}$, et al. Serum exosomal miR-210 as a potential biomarker for clear cell renal cell carcinoma. J Cell Biochem. 2018. doi:10.1002/jcb.27347

41. von Brandenstein M, Pandarakalam JJ, Kroon L, et al. MicroRNA 15a, inversely correlated to PKCalpha, is a potential marker to differentiate between benign and malignant renal tumors in biopsy and urine samples. Am J Pathol. 2012;180(5):1787-1797. doi:10.1016/j.ajpath.2012.01.014 
42. Shinden Y, Akiyoshi S, Ueo H, et al. Diminished expression of MiR-15a is an independent prognostic marker for breast cancer cases. Anticancer Res. 2015;35(1):123-127.

43. Zhu K, He Y, Xia C, et al. MicroRNA-15a inhibits proliferation and induces apoptosis in CNE1 nasopharyngeal carcinoma cells. Oncol Res. 2016;24(3):145-151. doi:10.3727/ 096504016X14611963142290

44. Li G, Zhao A, Peoch M, et al. Detection of urinary cell-free miR-210 as a potential tool of liquid biopsy for clear cell renal cell carcinoma. Urol Oncol. 2017;35(5):294-299. doi:10.1016/j. urolonc.2016.12.007

45. Petrozza V, Pastore AL, Palleschi G, et al. Secreted miR-210-3p as non-invasive biomarker in clear cell renal cell carcinoma. Oncotarget. 2017;8(41):69551-69558. doi:10.18632/ oncotarget. 18449

46. Petrozza V, Costantini M, Tito C, et al. Emerging role of secreted miR-210-3p as potential biomarker for clear cell renal cell carcinoma metastasis. Cancer Biomark. 2020;27(2):181-188. doi:10.3233/CBM-190242

47. Di Meo A, Brown MD, Finelli A, et al. Prognostic urinary miRNAs for the assessment of small renal masses. Clin Biochem. 2020;75:15-22. doi:10.1016/j.clinbiochem.2019.10.002.

48. Wang C, Cai L, Liu J, et al. MicroRNA-30a-5p inhibits the growth of renal cell carcinoma by modulating GRP78 expression. Cell Physiol Biochem. 2017;43(6):2405-2419. doi: $10.1159 / 000484394$

49. Zheng B, Zhu H, Gu D, et al. MiRNA-30a-mediated autophagy inhibition sensitizes renal cell carcinoma cells to sorafenib. Biochem Biophys Res Commun. 2015;459(2):234-239. doi:10.1016/j.bbrc.2015.02.084

50. Outeiro-Pinho G, Barros-Silva D, Aznar E, et al. MicroRNA-30a$5 \mathrm{p}(\mathrm{me})$ : a novel diagnostic and prognostic biomarker for clear cell renal cell carcinoma in tissue and urine samples. J Exp Clin Cancer Res. 2020;39(1):98. doi:10.1186/s13046-020-01600-3

51. Chen X, Ba Y, Ma L, et al. Characterization of microRNAs in serum: a novel class of biomarkers for diagnosis of cancer and other diseases. Cell Res. 2008;18(10):997-1006. doi:10.1038/cr.2008.282

52. Tusong H, Maolakuerban N, Guan J, et al. Functional analysis of serum microRNAs miR-21 and miR-106a in renal cell carcinoma. Cancer Biomark. 2017;18(1):79-85. doi:10.3233/CBM-160676

53. Huang G, Li H, Wang J, et al. Combination of tumor suppressor miR-20b-5p, miR-30a-5p, and miR-196a-5p as a serum diagnostic panel for renal cell carcinoma. Pathol Res Pract. 2020;216 (11):153152. doi:10.1016/j.prp.2020.153152

54. Heinemann FG, Tolkach Y, Deng M, et al. Serum miR-122-5p and miR-206 expression: non-invasive prognostic biomarkers for renal cell carcinoma. Clin Epigenetics. 2018;10(1):11. doi:10.1186/s13148-018-0444-9

55. Liu S, Deng X, Zhang J. Identification of dysregulated serum miR-508-3p and miR-885-5p as potential diagnostic biomarkers of clear cell renal carcinoma. Mol Med Rep. 2019;20 (6):5075-5083. doi:10.3892/mmr.2019.10762

56. Zhao L, Liu K, Pan X, et al. miR-625-3p promotes migration and invasion and reduces apoptosis of clear cell renal cell carcinoma. Am J Transl Res. 2019;11(10):6475-6486.

57. Pigati L, Yaddanapudi SCS, Iyengar R, et al. Selective release of microRNA species from normal and malignant mammary epithelial cells. PLoS One. 2010;5(10):e13515. doi:10.1371/journal. pone. 0013515

58. Wang K, Yuan $\mathrm{Y}$, Cho JH, et al. Comparing the MicroRNA spectrum between serum and plasma. PLoS One. 2012;7(7): e41561. doi:10.1371/journal.pone.0041561

59. Lou N, Ruan AM, Qiu B, et al. miR-144-3p as a novel plasma diagnostic biomarker for clear cell renal cell carcinoma. Urol Oncol. 2017;35(1):36 e37-36 e14. doi:10.1016/j. urolonc.2016.07.012
60. Chanudet E, Wozniak MB, Bouaoun L, et al. Large-scale genome-wide screening of circulating microRNAs in clear cell renal cell carcinoma reveals specific signatures in late-stage disease. Int $J$ Cancer. 2017;141(9):1730-1740. doi:10.1002/ ijc. 30845

61. Wang XG, Zhu YW, Wang T, et al. MiR-483-5p downregulation contributed to cell proliferation, metastasis, and inflammation of clear cell renal cell carcinoma. Kaohsiung J Med Sci. 2020. doi: $10.1002 / \mathrm{kjm} 2.12320$

62. Lucca I, de Martino M, Hofbauer SL, et al. Comparison of the prognostic value of pretreatment measurements of systemic inflammatory response in patients undergoing curative resection of clear cell renal cell carcinoma. World J Urol. 2015;33 (12):2045-2052. doi:10.1007/s00345-015-1559-7

63. Ohno Y, Nakashima J, Ohori M, et al. Followup of neutrophil-tolymphocyte ratio and recurrence of clear cell renal cell carcinoma. J Urol. 2012;187(2):411-417. doi:10.1016/j.juro.2011.10.026

64. Sonoda Y, Warita M, Suzuki T, et al. Proteolipid protein 2 is associated with melanoma metastasis. Oncol Rep. 2010;23 (2):371-376.

65. Xiao W, Wang C, Chen K, et al. MiR-765 functions as a tumour suppressor and eliminates lipids in clear cell renal cell carcinoma by downregulating PLP2. EBioMedicine. 2020;51:102622. doi:10.1016/j.ebiom.2019.102622

66. Chen X, Lou N, Ruan A, et al. miR-224/miR-141 ratio as a novel diagnostic biomarker in renal cell carcinoma. Oncol Lett. 2018;16 (2):1666-1674. doi:10.3892/ol.2018.8874

67. Valadi H, Ekstrom K, Bossios A, et al. Exosome-mediated transfer of mRNAs and microRNAs is a novel mechanism of genetic exchange between cells. Nat Cell Biol. 2007;9(6):654-659. doi: $10.1038 /$ ncb 1596

68. Honegger A, Schilling D, Bastian S, et al. Dependence of intracellular and exosomal microRNAs on viral E6/E7 oncogene expression in HPV-positive tumor cells. PLoS Pathog. 2015;11 (3):e1004712. doi:10.1371/journal.ppat.1004712

69. Kahlert C, Melo SA, Protopopov A, et al. Identification of double-stranded genomic DNA spanning all chromosomes with mutated KRAS and p53 DNA in the serum exosomes of patients with pancreatic cancer. J Biol Chem. 2014;289(7):3869-3875. doi:10.1074/jbc.C113.532267

70. Bao L, You B, Shi S, et al. Metastasis-associated miR-23a from nasopharyngeal carcinoma-derived exosomes mediates angiogenesis by repressing a novel target gene TSGA10. Oncogene. 2018;37(21):2873-2889. doi:10.1038/s41388-018-0183-6

71. Keller S, Sanderson MP, Stoeck A, et al. Exosomes: from biogenesis and secretion to biological function. Immunol Lett. 2006;107 (2):102-108. doi:10.1016/j.imlet.2006.09.005

72. Rana S, Malinowska K, Zoller M. Exosomal tumor microRNA modulates premetastatic organ cells. Neoplasia. 2013;15 (3):281-295. doi:10.1593/neo.122010

73. Zhang L, Zhang S, Yao J, et al. Microenvironment-induced PTEN loss by exosomal microRNA primes brain metastasis outgrowth. Nature. 2015;527(7576):100-104. doi:10.1038/nature15376

74. Li Y, Zheng Q, Bao C, et al. Circular RNA is enriched and stable in exosomes: a promising biomarker for cancer diagnosis. Cell Res. 2015;25(8):981-984. doi:10.1038/cr.2015.82

75. Butz H, Nofech-Mozes R, Ding Q, et al. Exosomal MicroRNAs are diagnostic biomarkers and can mediate cell-cell communication in renal cell carcinoma. Eur Urol Focus. 2016;2(2):210-218. doi:10.1016/j.euf.2015.11.006

76. Yang C, Robbins PD. The roles of tumor-derived exosomes in cancer pathogenesis. Clin Dev Immunol. 2011;2011:842849. doi:10.1155/2011/842849

77. Zhang W, Ni M, Su Y, et al. MicroRNAs in serum exosomes as potential biomarkers in clear-cell renal cell carcinoma. Eur Urol Focus. 2018;4(3):412-419. doi:10.1016/j.euf.2016.09.007 
78. Folkerts H, Wierenga AT, van den Heuvel FA, et al. Elevated VMP1 expression in acute myeloid leukemia amplifies autophagy and is protective against venetoclax-induced apoptosis. Cell Death Dis. 2019;10(6):421. doi:10.1038/s41419-019-1648-4

79. Ying Q, Liang L, Guo W, et al. Hypoxia-inducible microRNA-210 augments the metastatic potential of tumor cells by targeting vacuole membrane protein 1 in hepatocellular carcinoma. Hepatology. 2011;54(6):2064-2075. doi:10.1002/ hep. 24614

80. Crentsil VC, Liu H, Sellitti DF. Comparison of exosomal microRNAs secreted by 786-O clear cell renal carcinoma cells and HK-2 proximal tubule-derived cells in culture identifies microRNA-205 as a potential biomarker of clear cell renal carcinoma. Oncol Lett. 2018;16(1):1285-1290. doi:10.3892/ ol.2018.8751

81. Xu XW, Li S, Yin F, et al. Expression of miR-205 in renal cell carcinoma and its association with clinicopathological features and prognosis. Eur Rev Med Pharmacol Sci. 2018;22 (3):662-670. doi:10.26355/eurrev_201802_14291

82. Koren E, Fuchs Y. The bad seed: cancer stem cells in tumor development and resistance. Drug Resist Updat. 2016;28:1-12. doi:10.1016/j.drup.2016.06.006

83. Vahidian F, Mohammadi H, Ali-Hasanzadeh $M$, et al. MicroRNAs and breast cancer stem cells: potential role in breast cancer therapy. J Cell Physiol. 2019;234(4):3294-3306. doi: $10.1002 /$ jcp. 27246

84. Wang L, Yang G, Zhao D, et al. CD103-positive CSC exosome promotes EMT of clear cell renal cell carcinoma: role of remote MiR-19b-3p. Mol Cancer. 2019;18(1):86. doi:10.1186/s12943019-0997-z

85. Song S, Long $\mathrm{M}, \mathrm{Yu} \mathrm{G}$, et al. Urinary exosome miR-30c-5p as a biomarker of clear cell renal cell carcinoma that inhibits progression by targeting HSPA5. J Cell Mol Med. 2019;23 (10):6755-6765. doi:10.1111/jcmm.14553

86. Yang GH, Li S, Pestka JJ. Down-regulation of the endoplasmic reticulum chaperone GRP78/BiP by vomitoxin (deoxynivalenol). Toxicol Appl Pharmacol. 2000;162(3):207-217. doi:10.1006/ taap. 1999.8842

87. Xiao CT, Lai WJ, Zhu WA, et al. MicroRNA derived from circulating exosomes as noninvasive biomarkers for diagnosing renal cell carcinoma. Onco Targets Ther. 2020;13:10765-10774. doi:10.2147/OTT.S271606

88. Sun B, Zhang J, Liu M, et al. Alkannin inhibits proliferation, migration and invasion of hepatocellular carcinoma cells via regulation of miR-92a. Biomed Pharmacother. 2019;114:108782. doi:10.1016/j.biopha.2019.108782

89. Si H, Sun X, Chen Y, et al. Circulating microRNA-92a and microRNA-2 1 as novel minimally invasive biomarkers for primary breast cancer. J Cancer Res Clin Oncol. 2013;139 (2):223-229. doi:10.1007/s00432-012-1315-y

90. Shi Y, Liu Z. Serum miR-92a-1 is a novel diagnostic biomarker for colorectal cancer. J Cell Mol Med. 2020;24(15):8363-8367. doi: $10.1111 / \mathrm{jcmm} .15282$

91. Cui Y, Yang J, Bai Y, et al. miR-424-5p regulates cell proliferation and migration of esophageal squamous cell carcinoma by targeting SIRT4. J Cancer. 2020;11(21):6337-6347. doi:10.7150/ jca. 50587

92. Liu X, Fu Y, Zhang G, et al. miR-424-5p promotes anoikis resistance and lung metastasis by inactivating hippo signaling in thyroid cancer. Mol Ther Oncol. 2019;15:248-260. doi:10.1016/j. omto.2019.10.008

93. Jin L, Li Y, Liu J, et al. Tumor suppressor miR-149-5p is associated with cellular migration, proliferation and apoptosis in renal cell carcinoma. Mol Med Rep. 2016;13(6):5386-5392. doi: $10.3892 / \mathrm{mmr} .2016 .5205$
94. Guo P, Sheng M, Liu H, et al. Effects of miR-218-1-3p and miR-149 on proliferation and apoptosis of non-small cell lung cancer cells. Oncol Lett. 2020;20(4):96. doi:10.3892/ ol.2020.11957

95. Youssef YM, White NM, Grigull J, et al. Accurate molecular classification of kidney cancer subtypes using microRNA signature. Eur Urol. 2011;59(5):721-730. doi:10.1016/j. eururo.2011.01.004

96. Qi Y, Wang L, Wang K, et al. New mechanistic insights of clear cell renal cell carcinoma from integrated miRNA and mRNA expression profiling studies. Biomed Pharmacother. 2019;111:821-834. doi:10.1016/j.biopha.2018.12.099

97. Nie W, Ni D, Ma X, et al. miR122 promotes proliferation and invasion of clear cell renal cell carcinoma by suppressing Forkhead box O3. Int $J$ Oncol. 2019;54(2):559-571. doi:10.3892/ijo.2018.4636

98. Lin $\mathrm{C}$, Li Z, Chen $\mathrm{P}$, et al. Oncogene miR-154-5p regulates cellular function and acts as a molecular marker with poor prognosis in renal cell carcinoma. Life Sci. 2018;209:481-489. doi:10.1016/j.lfs.2018.08.044

99. Zhang J, Ye Y, Chang DW, et al. Global and targeted miRNA expression profiling in clear cell renal cell carcinoma tissues potentially links miR-155-5p and miR-210-3p to both tumorigenesis and recurrence. Am J Pathol. 2018;188(11):2487-2496. doi:10.1016/j.ajpath.2018.07.026

100. Ralla B, Busch J, Florcken A, et al. miR-9-5p in nephrectomy specimens is a potential predictor of primary resistance to first-line treatment with tyrosine kinase inhibitors in patients with metastatic renal cell carcinoma. Cancers (Basel). 2018;10 (9):321. doi:10.3390/cancers 10090321

101. Garrigos C, Molina-Pinelo S, Melendez R, et al. MicroRNAs as potential predictors of extreme response to tyrosine kinase inhibitors in renal cell cancer. Urol Oncol. 2020;38(7):640 e623-640 e629. doi:10.1016/j.urolonc.2020.01.012

102. Incorvaia L, Fanale D, Badalamenti G, et al. A "lymphocyte MicroRNA signature" as predictive biomarker of immunotherapy response and plasma pd-1/pd-11 expression levels in patients with metastatic renal cell carcinoma: pointing towards epigenetic reprogramming. Cancers (Basel). 2020;12(11):3396. doi:10.3390/cancers 12113396

103. Qu F, Ye J, Pan X, et al. MicroRNA-497-5p down-regulation increases PD-L1 expression in clear cell renal cell carcinoma. J Drug Target. 2019;27(1):67-74. doi:10.1080/ 1061186X.2018.1479755

104. Tito C, De Falco E, Rosa P, et al. Circulating microRNAs from the molecular mechanisms to clinical biomarkers: a focus on the clear cell renal cell carcinoma. Genes (Basel). 2021;12(8):1154. doi:10.3390/genes 12081154

105. Szabo Z, Szegedi K, Gombos K, et al. Expression of miRNA-21 and miRNA-221 in clear cell renal cell carcinoma (ccRCC) and their possible role in the development of ccRCC. Urol Oncol. 2016;34(12):533 e521-533 e527. doi:10.1016/j. urolonc.2016.06.011

106. Liu F, Chen N, Xiao R, et al. miR-144-3p serves as a tumor suppressor for renal cell carcinoma and inhibits its invasion and metastasis by targeting MAP3K8. Biochem Biophys Res Commun. 2016;480(1):87-93. doi:10.1016/j.bbrc.2016.10.004

107. Liep J, Kilic E, Meyer HA, et al. Cooperative effect of miR-141$3 p$ and miR-145-5p in the regulation of targets in clear cell renal cell carcinoma. PLoS One. 2016;11(6):e0157801. doi:10.1371/ journal.pone. 0157801

108. Chen B, Duan L, Yin G, et al. Simultaneously expressed miR-424 and miR-381 synergistically suppress the proliferation and survival of renal cancer cells - Cdc2 activity is up-regulated by targeting WEE1. Clinics (Sao Paulo). 2013;68(6):825-833. doi:10.6061/clinics/2013(06)17 
109. He Y, Liu J, Wang Y, et al. Role of miR-486-5p in regulating renal cell carcinoma cell proliferation and apoptosis via TGF-beta-activated kinase 1. J Cell Biochem. 2019;120 (3):2954-2963. doi:10.1002/jcb.26900
110. Yao D, Xia S, Jin C, et al. Feedback activation of GATA1/ miR-885-5p/PLIN3 pathway decreases sunitinib sensitivity in clear cell renal cell carcinoma. Cell Cycle. 2020;19 (17):2195-2206. doi:10.1080/15384101.2020.1801189

\section{Publish your work in this journal}

Cancer Management and Research is an international, peer-reviewed open access journal focusing on cancer research and the optimal use of preventative and integrated treatment interventions to achieve improved outcomes, enhanced survival and quality of life for the cancer patient.
The manuscript management system is completely online and includes a very quick and fair peer-review system, which is all easy to use. Visit http://www.dovepress.com/testimonials.php to read real quotes from published authors. 